\title{
SOX2 has a crucial role in the lineage determination and proliferation of mesenchymal stem cells through Dickkopf-1 and C-MYC
}

\author{
SB Park ${ }^{1,2,5}, K_{W}$ Seo $^{1,2,3,5}$, AY So $^{1,2}$, MS Seo $^{1,2}, \mathrm{KR} \mathrm{Yu}^{1,2}, \mathrm{SK} \mathrm{Kang}^{4}$ and KS Kang ${ }^{\star, 1,2,3}$
}

SOX2 is a well-known core transcription factor in embryonic stem cells (ESCs) and has an important role in the maintenance of pluripotency. Recently, SOX2 expression has also been reported in adult stem cells (ASCs), but the role of SOX2 in ASCs remains unknown. In this study, we examined the molecular mechanisms of SOX2 in human mesenchymal stem cells (hMSCs), a type of ASCs, by performing inhibition studies. SOX2 inhibition resulted in altered cell growth and differentiation capabilities. These changes coincided with a decrease in Dickkopf-1 (DKK1), a soluble inhibitor of WNT signaling. Chromatin immunoprecipitation and luciferase assays showed that SOX2 binds to DKK1 and has a positive regulatory role in transcription. The enforced expression of DKK1 in SOX2-inhibited hMSCs reversed the differentiation deformities, but could not abrogate the cell proliferation defect. Proliferation was regulated by C-MYC, whose expression can also be controlled by SOX2. Our study shows that SOX2 directly regulates DKK1 expression and, as a consequence, determines the differentiation lineage of hMSCs. Moreover, SOX2 also regulates proliferation by affecting c-MYC. Therefore, these results suggest that SOX2 might have a specific function by regulating DKK1 and c-MYC in the differentiation and growth of ASCs, which is separate from its roles in ESCs.

Cell Death and Differentiation (2012) 19, 534-545; doi:10.1038/cdd.2011.137; published online 21 October 2011

The signaling pathways in the core transcriptional network of embryonic stem cells (ESCs) have previously been well described. ${ }^{1}$ Several core ESC transcription factors are also expressed in human mesenchymal stem cells (hMSCs). ${ }^{2,3}$ Octamer-binding transcription factor 4 (OCT4) is a POU domain-containing transcription factor, known to be essential for the stemness and pluripotency of ESCs. ${ }^{4}$ OCT4A has conserved roles in ESCs and in human umbilical cord bloodderived MSCs (hUCB-MSCs). ${ }^{5}$ OCT4 interacts with sexdetermining region $\mathrm{Y}$-box 2 (SOX2), and genome-wide mapping of OCT4- and SOX2-binding sites in human ESCs shows that they co-target multiple genes. ${ }^{6}$ The cis-regulatory element to which the SOX2-OCT4 complex is bound consists of neighboring SOX (5'-CATTGTT-3') and OCT (5'-ATGCAA AT- $3^{\prime}$ ) elements. ${ }^{7}$ Indeed, SOX2 was reported to be indispensable for the maintenance of ESC pluripotency, ${ }^{8}$ and ESCs from Sox2-null mice primarily differentiate into trophectoderm-like cells. ${ }^{9}$ Microarray analysis has shown that SOX2 regulates multiple transcription factors that affect the OCT4 expression. ${ }^{10}$ SOX2 expression has been detected in ESCs and in several tissues derived from hMSCs, including adipose tissue, dermis, heart, ${ }^{2}$ and neural tissue. ${ }^{11}$ An integrated expression profile and chromatin immunoprecipitation (ChIP)sequencing analysis showed that SOX2 is involved in the
BMP signaling pathway, steroid metabolic processes, histone modifications, and many receptor-mediated signaling pathways, such as IGF1R and ITPR2. ${ }^{12}$ The well-known oncogene c-MYC is also an important transcription factor in ESCs. Many genes are regulated by c-MYC and consequently induce cell proliferation. Deregulation of C-MYC contributes to the genesis of most human tumors. ${ }^{13}$ Our previous study has shown that c-MYC is closely connected with the proliferation and epigenetic regulation of hMSCs. ${ }^{14}$ Moreover, a recent study has demonstrated a hypothetical relationship between c-MYC and SOX2. ${ }^{15}$

WNT genes, a family of 19 genes in humans and mice, produce secreted proteins that are involved in cell proliferation, differentiation, and apoptosis. These genes are also crucial for embryonic tissue development and adult tissue regeneration. ${ }^{16}$ Canonical WNT/ $\beta$-CATENIN signaling is important for the stemness and differentiation of stem cells. ${ }^{17}$ The addition of Dickkopf-1 (DKK1), an antagonist of WNT signaling, allows hMSCs to re-enter the cell cycle by inhibiting the canonical WNT/ $\beta$-CATENIN-signaling pathway. ${ }^{18}$ The secretion of DKK1 by hMSCs inhibits the growth of breast cancer cells. ${ }^{19}$ The presence of DKK1 triggers a disruption in the canonical WNT cascade and results in the inhibition of hMSC osteogenesis. ${ }^{20}$ DKK1 is transiently upregulated in the

\footnotetext{
${ }^{1}$ Adult Stem Cell Research Center, College of Veterinary Medicine, Seoul National University, Seoul, Republic of Korea; ${ }^{2}$ Laboratory of Stem Cell and Tumor Biology, Department of Veterinary Public Health, College of Veterinary Medicine, Seoul National University, Seoul, Republic of Korea; ${ }^{3}$ Research Institute for Veterinary Science, College of Veterinary Medicine, Seoul National University, Seoul, Republic of Korea and ${ }^{4}$ Department of Veterinary Biotechnology, Laboratory of Veterinary Biotechnology, College of Veterinary Medicine and BK21Program for Veterinary Science, Seoul National University, Seoul, Republic of Korea

${ }^{*}$ Corresponding author: KS Kang, Adult Stem Cell Research Center, College of Veterinary Medicine, Seoul National University, Seoul 151-742, Republic of Korea. Tel: + 822880 1298; Fax: + 822876 7610; E-mail: kangpub@snu.ac.kr

${ }^{5}$ These authors contributed equally to this work.
}

Keywords: SOX2; DKK1; WNT; C-MYC; mesenchymal stem cell

Abbreviations: ESC, embryonic stem cell; MSC, mesenchymal stem cell; ASC, adult stem cell

Received 18.7.11; revised 14.9.11; accepted 14.9.11; Edited by R De Maria; published online 21.10.11 
early stages of hMSC adipogenesis, which indicates that the WNT cascade is important for the adipogenic differentiation of hMSCs. ${ }^{21}$ SOX factors appear to modulate $\beta$-CATENIN/TCF activity through a variety of mechanisms, including proteinprotein interactions, DNA binding, recruitment of cofactors, and protein stability. ${ }^{22}$ SOX2 and $\beta$-CATENIN act synergistically in the transcriptional regulation of CCND1 in breast cancer cells. ${ }^{23}$ Recent studies have demonstrated that SOX2 contributes to the inhibition of WNT signaling and regulates osteoblast differentiation. ${ }^{24,25}$ However, SOX2 does not bind to TCF/LEF in human cells (Ingenuity Systems, Redwood City, CA, USA), and how WNT signaling is regulated by SOX2 is still unknown.

Previous studies have shown that SOX2 is expressed in several types of adult stem cells (ASCs), including neural stem cells (NSCs), ${ }^{26}$ dermal stem cells, ${ }^{27}$ and germline stem cells. ${ }^{28}$ However, the only known role of SOX2 is in NSCs, and relates to the self-renewal and neuronal differentiation of NSCs. ${ }^{26}$ The transcriptomes of NSCs, ESCs, and ASCs are quite different from each other. ${ }^{29,30}$ Therefore, SOX2 may have different roles in each type of stem cell.

\section{Results}

SOX2 is expressed in hUCB-MSCs, and cell growth is altered after SOX2 inhibition in hUCB-MSCs. To investigate the role of SOX2 in hMSCs, we examined SOX2 expression. Three well-known types of hMSCs (hUCB-MSCs, human adipose tissue-derived MSCs (hADMSCs), and human bone-marrow derived MSCs (hBMMSCs)) were tested. Tera-1, an embryonic carcinoma cell line, was used as a positive control for SOX2 expression. SOX2 expression was found in hUCB-MSCs, although the levels were lower than in the tera-1 cells (Figure 1a). To quantify the SOX2 expression levels in hMSCs, quantitative reverse transcription PCR (RT-PCR) was performed (Figure 1b). hUCB-MSCs exhibited higher SOX2 expression than the other hMSCs analyzed. Furthermore, immunocytochemistry confirmed the nuclear localization of SOX2 in tera-1 cells and hUCB-MSCs (Figure 1c). Fibronectin staining was performed to determine the morphology of the cells. In the tera-1 cells, SOX2 was highly expressed in the nucleus and colocalized with Hoechst. SOX2 expression was also detected in the nuclear region of hUCB-MSCs (Supplementary Figure 1a). However, no detectable signals were observed in the nucleus or cytoplasm of hAD-MSCs or hBM-MSCs. Because SOX2 expression in hAD- and hBM-MSCs was so low, we confirmed whether all three types of hMSCs retained pluripotency. Western blotting for SOX2 and other stem cell markers such as OCT4 and C-MYC was performed using nuclear and cytoplasmic extracts (Supplementary Figure 1b). Although the levels were lower than in hUCBMSCs, SOX2 and OCT4 could be detected in hAD- and hBM-MSCs.

To investigate the role of SOX2, knockdown was performed in hUCB-MSCs using short hairpin RNA-producing lentivirus. The sh-SOX2 construct targets the $3^{\prime}$-untranslated region of SOX2. A non-targeting, random sequence-inserted lentivirus was used as a control. SOX2 knockdown (sh-SOX2) in
hUCB-MSCs caused a decrease in cell proliferation compared with the sh-control (Figures 1d and g). These proliferation rates correlated with SOX2 expression levels after lentiviral infection (Figure 1e). The cell cycle was also analyzed by fluorescence-activated cell sorting (FACS) in both the sh-SOX2and sh-control-treated cells (Figure 1f). After sh-SOX2 treatment, the proportion of cells in G0/G1 increased, and the portion of cells in $S$ phase decreased, compared with the sh-control. To confirm this phenotype, another SOX2 knockdown study was designed using a commercially available, specific siRNA for SOX2 inhibition (si-SOX2) and a nontargeting random sequence-inserted siRNA as a control (si-control). At $48 \mathrm{~h}$ after siRNA transfection, the cells treated with si-SOX2 displayed growth retardation compared with si-control-treated cells (Supplementary Figure 2a). By FACS analysis and MTT assays, the si-SOX2 treatment caused a decrease in S-phase composition (Supplementary Figure 2c) and a decrease in the proliferation rate (Supplementary Figure 2d).

Differentiation ability is altered after SOX2 inhibition. To validate the role of SOX2 in the differentiation of hUCBMSCs, adipogenic, osteogenic, and chondrogenic differentiation studies were performed. After 2 weeks of adipogenic induction, a decreased number of oil droplets were found in sh-SOX2 compared with the sh-control-treated cells (Figure 2a). The optical density of oil red $\mathrm{O}$ after elution confirmed the morphological observations (Figure $2 b$ ). Molecular markers of adipogenic differentiation, PPAR- $\gamma$ and $C / E B P-\beta$, were analyzed by RT-PCR (Figure $2 \mathrm{c}$ ). The relative expression of $P P A R-\gamma$ and $C / E B P-\beta$ decreased in sh-SOX2 cells, compared with their levels in sh-control cells. An adipogenic differentiation assay was also conducted using cells transiently inhibited with siRNAs. Differentiation was initiated $48 \mathrm{~h}$ after of siRNA transfection and continued for 2 weeks. The cells treated with si-SOX2 manifested a decreased level of lipid droplet staining (Supplementary Figures $3 a-c)$. However, the decrease in adipogenesis was greater in the hUCB-MSCs treated with the sh-SOX2 lentivirus. There was no difference in the elution of oil red 0 between the si-control and si-SOX2 conditions (Supplementary Figure 3b). The molecular markers of adipogenic differentiation were decreased. These results show that the long-term inhibition of SOX2 by lentivirus led to more severe effects on the adipogenesis of hUCB-MSCs than transient inhibition with siRNA.

After 2 weeks of osteogenic induction, alizarin red $S$ staining was performed (Figures $2 d$ and e). In contrast to adipogenesis, the osteogenic differentiation ability was increased by the sh-SOX2 treatment, compared with the sh-control. The expression of osteogenic molecular markers, COL $1 A 2$ and VDR, was increased in sh-SOX2 compared with sh-control-treated cells (Figure 2f). In the transient SOX2 inhibition study, there were small differences between the si-control and si-SOX2 conditions after 2 weeks of osteogenic differentiation. Alizarin red $S$ staining was nearly identical (Supplementary Figures 3d-f), but the levels of COL1A2 and $V D R$ were increased in si-SOX2-treated cells compared with the si-control (Supplementary Figure $3 \mathrm{f}$ ).

After 2 weeks of chondrogenic induction, hUCB-MSCs aggregated and formed a pellet (Figure $2 \mathrm{~g}$ ). The pellet formed 
a

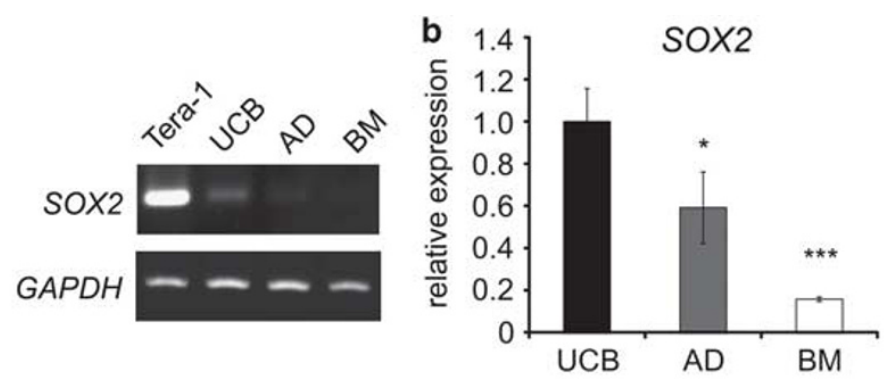

c
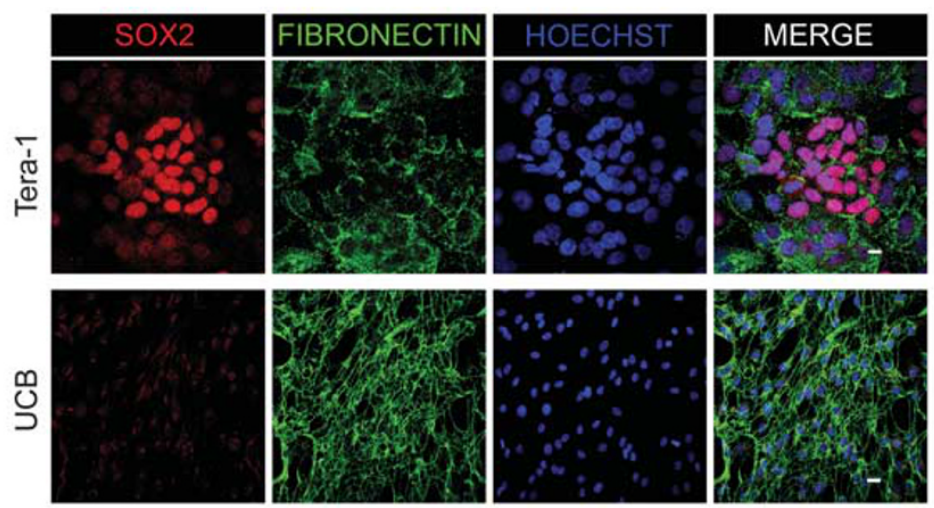

운
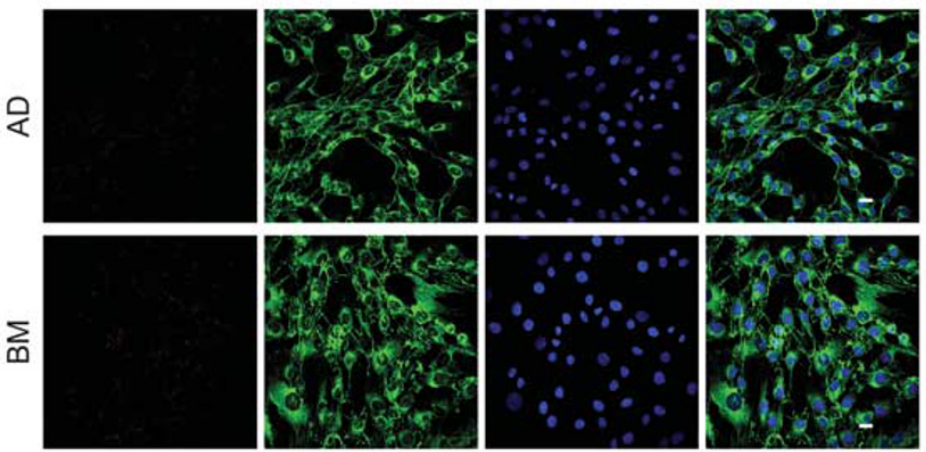

d
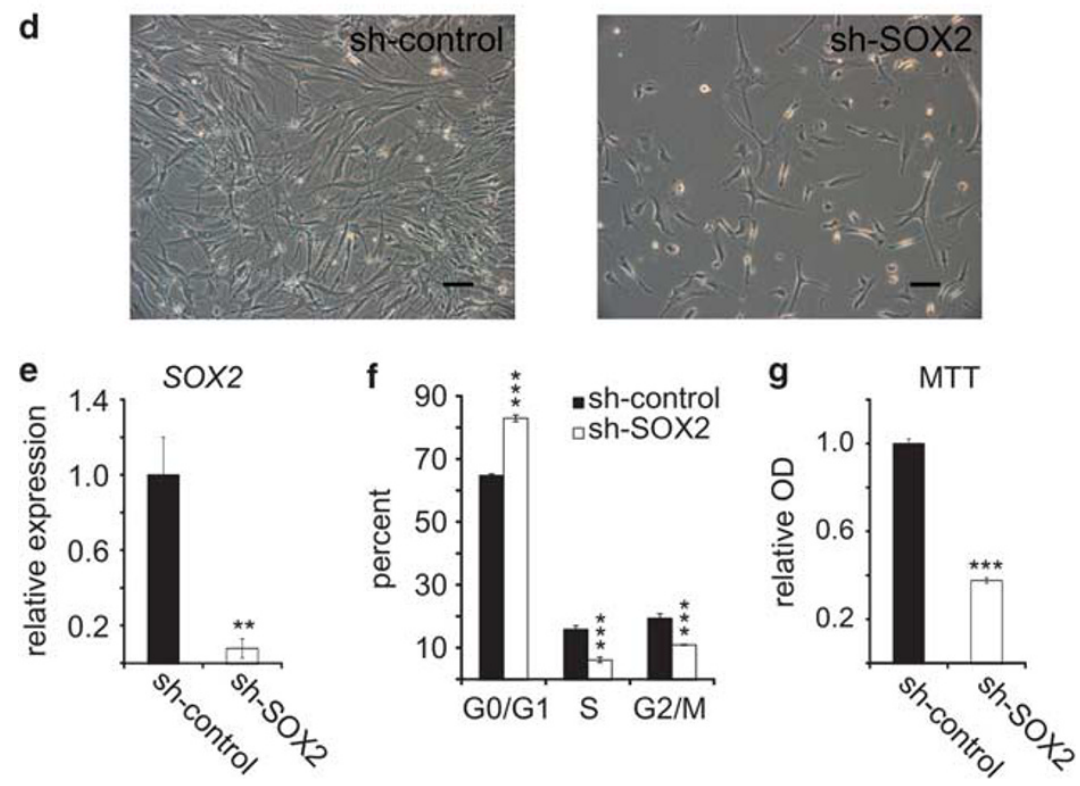
by the sh-SOX2-treated cells was larger than the sh-control. For histological evaluation, the pellets were cut into serial $3 \mu \mathrm{m}$ sections and stained with toluidine blue (Figure $2 \mathrm{~h}$ ). Toluidine blue-stained sections showed that sh-SOX2 cells formed larger pellets than the sh-control. The expression of chondrogenic markers, SOX9 and AGGRECAN, was increased in sh-SOX2 compared with the sh-control-treated cells (Figure 2i). The same results were obtained using transient siRNA inhibition as in the lentivirus-mediated inhibition study (Supplementary Figures $3 g-i$ ). These results a

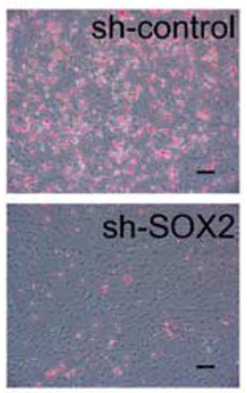

d
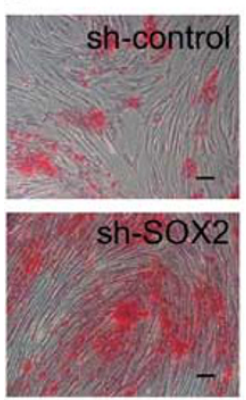

g
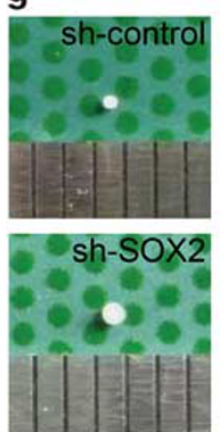

b

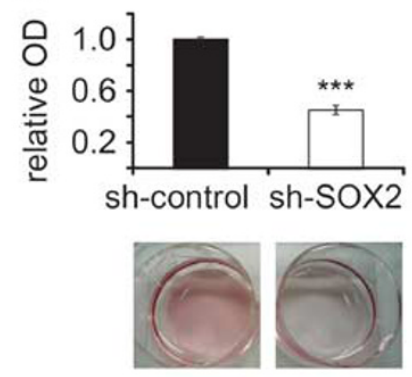

e

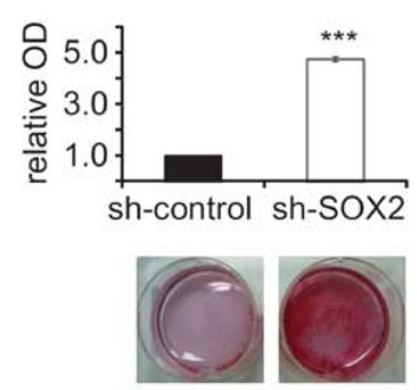

h

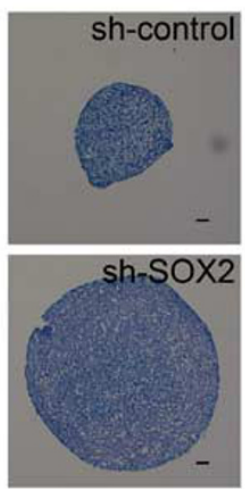

C

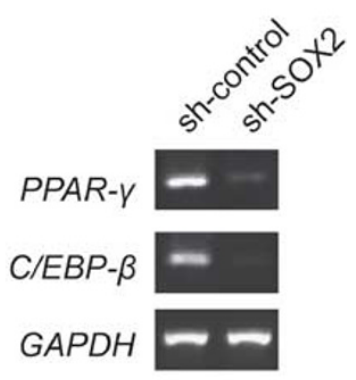

f

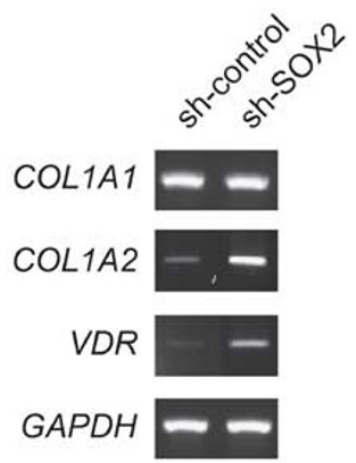

i

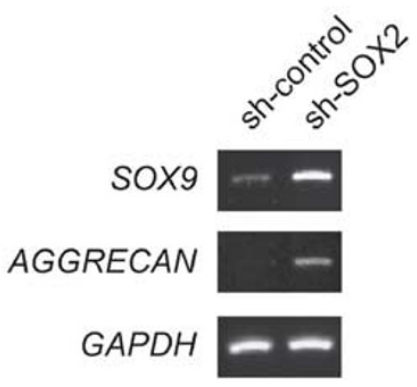

Figure 2 Differentiation abnormalities after SOX2 knockdown in hUCB-MSCs. (a-c) Adipogenic differentiation analysis after SOX2 knockdown with the lentiviral vector. (a) The number of oil red O-stained lipid droplets decreased after SOX2 knockdown. The scale bar represents $100 \mu \mathrm{m}$. (b) Elution of oil red $\mathrm{O}$ manifested reduced numbers of lipid droplets in SOX2-knockdown hUCB-MSCs. ${ }^{\star \star *} P<0.001$. (c) The expression of adipogenic markers decreased after SOX2 knockdown. (d-f) Osteogenic differentiation analysis after SOX2 knockdown with the lentiviral vector. (d) The alizarin red S-stained hUCB-MSC population increased after SOX2 knockdown. The scale bar represents $100 \mu \mathrm{m}$. (e) The elution of alizarin red S increased after SOX2 knockdown. ${ }^{* \star *} P<0.001$. (f) The expression of osteogenic markers increased after SOX2 knockdown. (g-i) Chondrogenic differentiation after SOX2 knockdown with the lentiviral vector. ( $\mathbf{g}$ and $\mathbf{h}$ ) The maximal diameters of sh-SOX2 cells were larger than those of the sh-control cells. The scale bar represents $100 \mu \mathrm{m}$. (i) The expression of chondrogenic markers increased after SOX2 knockdown

Figure 1 Analysis of SOX2 expression and proliferation in hMSCs. (a) RT-PCR of SOX2 in tera-1, hUCB-MSCs (UCB), hAD-MSCs (AD), and hBM-MSCs (BM). SOX2 expression in hUCB-MSCs was lower than in tera-1 cells. (b) Real-time PCR of SOX2 in hMSCs. The expression of SOX2 in hUCB-MSCs was higher than in other hMSCs. (c) Immunocytochemistry of SOX2. SOX2 expression in the nucleus was found in tera-1 cells and hUCB-MSCs. The scale bar represents $10 \mu \mathrm{m}$. (d) The hUCB-MSC population decreased after SOX2 knockdown. The scale bar represents $100 \mu \mathrm{m}$. (e) SOX2 expression decreased by 10\% of the sh-control value after SOX2 knockdown by lentivirus infection. (f) The proportion of cells in S and G0/G1 phase decreased and increased, respectively, after SOX2 knockdown. (g) Cell proliferation significantly decreased after SOX2 knockdown, as indicated by the MTT assays. ${ }^{*} P<0.05 ;{ }^{* \star} P<0.01 ;{ }^{* * *} P<0.001$ 
a

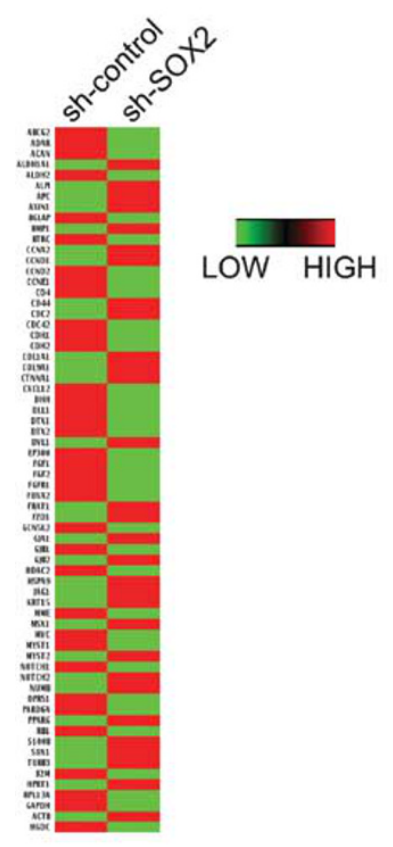

b
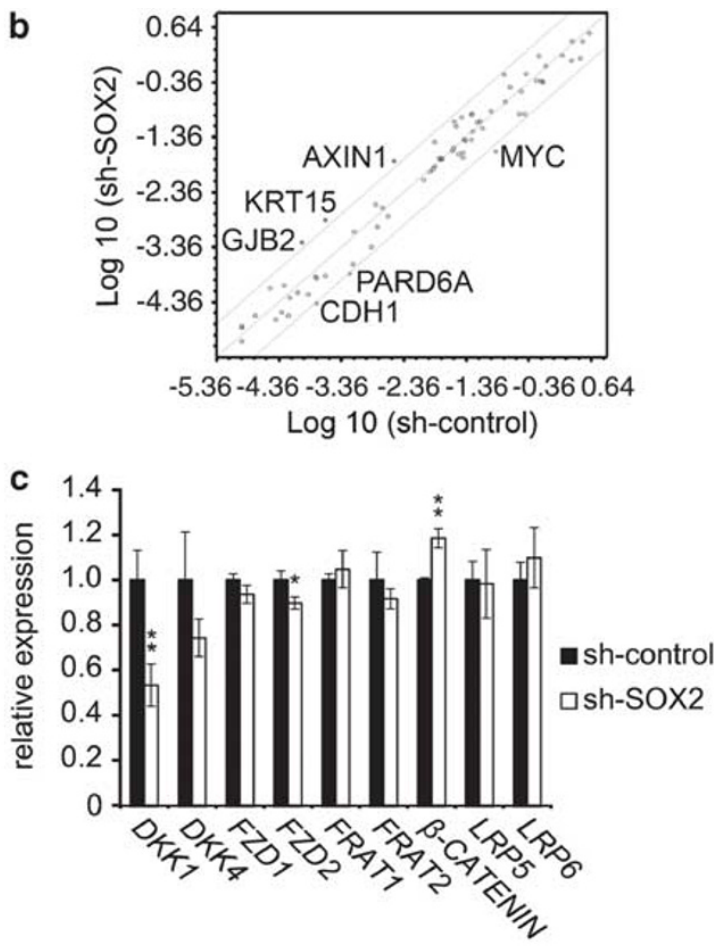

d

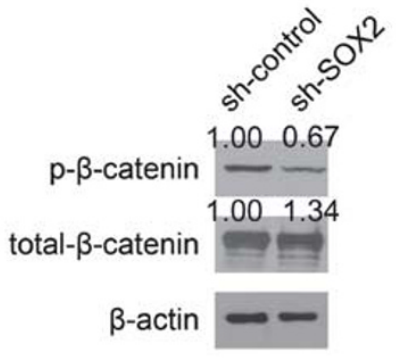

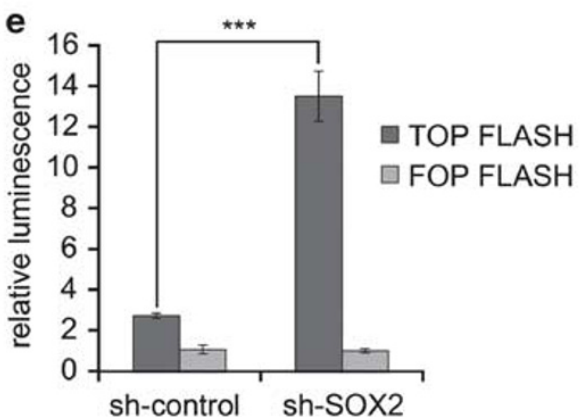

Figure 3 soX2 knockdown alter WNT signaling through DKK1. (a and $\mathbf{b})$ PCR array results. The expression of 88 frequently cited stem cell genes were compared between sh-control and sh-SOX2 cells. The expression of AXIN1, KRT15, and GJB2 increased by more than four-fold, and the expression of $c-M Y C, P A R D 6 A$, and CDH1 decreased by more than four-fold after SOX2 knockdown. (c) Real-time PCR analysis of WNT-related candidate genes. The expression of DKK1 was significantly decreased, but the expression of $\beta$-CATENIN was increased after SOX2 knockdown. ${ }^{*} P<0.05 ;{ }^{*} P<0.01$. (d) The western blot indicates reduced phosphorylated $\beta$-CATENIN ( $\mathrm{p}-\beta$-CATENIN) expression and increased total $\beta$-CATENIN (total- $\beta$-CATENIN) expression after SOX2 knockdown in hUCB-MSCs. Protein levels were normalized to $\beta$-ACTIN using Image $J$ analysis software. (e) The TOP/FOP Flash assay shows increased WNT signaling in sh-SOX2 cells. ${ }^{* \star} P<0.001$

demonstrated that SOX2 inhibition accelerated osteogenesis and chondrogenesis in hUCB-MSCs.

DKK1 expression decreases in SOX2-inhibited hUCBMSCs. To determine the molecular targets of SOX2 in hUCB-MSCs, a PCR array was performed (Figures $3 a, b$ and Supplementary Table 2). The expression of the 88 most-cited genes in stem cells was surveyed. Among them, the expression of $A X I N 1$ (one of the canonical $W N T / \beta$ CATENIN-signaling genes) was increased by 5.2 -fold after sh-SOX2 treatment, compared with the sh-control. To understand the regulation of WNT-signaling genes by SOX2 in greater detail, the levels of key components of the WNT signaling pathway (DKK1, DKK4, FZD1, FZD2, FRAT1, FRAT2, $\beta$-CATENIN, LRP5, and LRP6) were examined (Figure 3c). The levels of most WNT signalingrelated genes did not change or were only slightly altered after sh-SOX2 treatment, compared with the sh-control. However, the most significant change was found in DKK1 expression; expression was significantly decreased by sh-SOX2 compared with the sh-control. The expression of $\beta$-CATENIN was increased by sh-SOX2 compared with the sh-control. Levels of phosphorylated $\beta$-CATENIN were reduced by sh-SOX2 compared with the sh-control (Figure 3d). To confirm the regulation of WNT by SOX2, a TOP/FOP FLASH assay was performed (Figure 3e). The sh-SOX2 treatment resulted in an eight-fold increase in TOP FLASH luminescence, compared with the sh-control. In case of transient inhibition, the expression levels of WNT signaling-related genes were similar to those observed upon lentivirus-mediated inhibition of SOX2 (Supplementary 
a

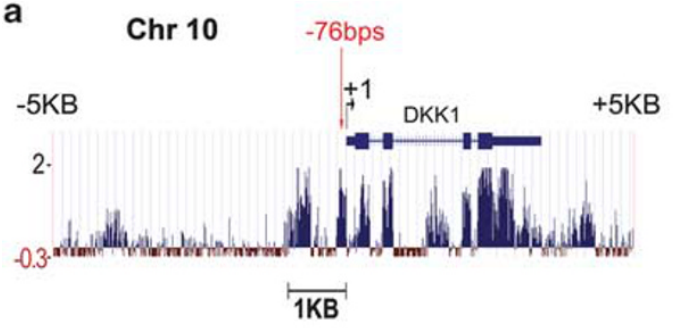

c

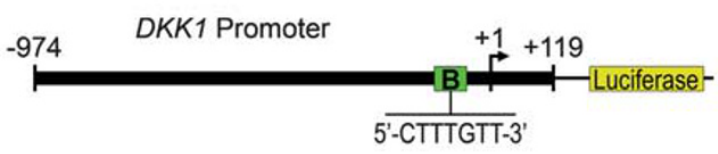

e
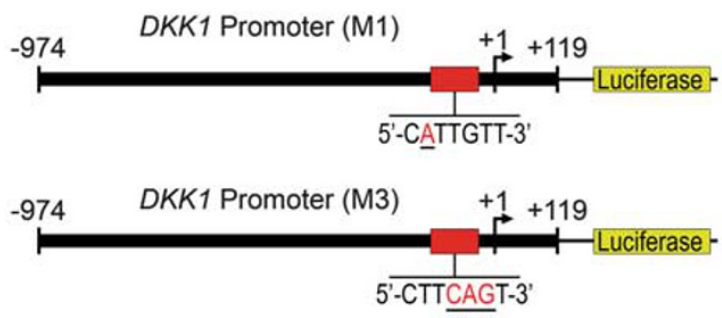

b

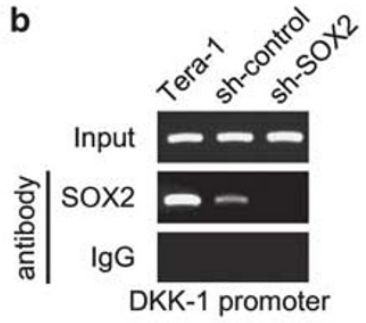

d

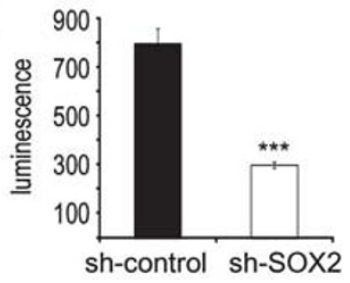

f
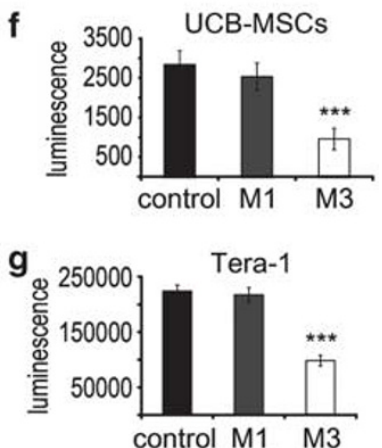

Figure 4 SOX2 directly binds to the DKK1 promoter region. (a) Sequence homology of region around DKK1 between mammals. Except for the exons of DKK1, the region $1 \mathrm{kbps}$ upstream of the transcriptional start site of $D K K 1$ was highly conserved and contained an HMG homeodomain ( $-76 \mathrm{bps})$. (b) ChIP assay for the $-76 \mathrm{bps}$ region of DKK1 showed SOX2-specific binding. (c) Luciferase reporter assay. Luciferase activity and transfection efficiency were normalized to transfection of the SV40 promoter control vector (SV40 promoter). The human DKK1 promoter region from $-974 \mathrm{bps}$ to $+119 \mathrm{bps}$ around the DKK1 transcription start site, which contains an HMG-binding domain, was cloned into the luciferase enhancer vector (DKK1 promoter). (d) The luciferase activity of sh-SOX2 was significantly decreased compared with that of the sh-control. ${ }^{* \star \star} P<0.001$. (e) Site-directed mutagenesis, involving single (M1) and triple (M3) mutations, was performed in the HMG domain of the DKK1 promoter vector. (f and g) The M1 (also SOX2 binding site) mutation had no effect on the luciferase activity, but the M3 mutation significantly reduced the luciferase activity in both hUCB-MSCs and tera-1 cells. ${ }^{* \star} P<0.001$

Figures $4 \mathrm{a}$ and $\mathrm{b})$. Moreover, the expression of TOP FLASH was increased by the si-SOX2 treatment (Supplementary Figure 4c). Therefore, $D K K 1$ is suggested as a strong candidate gene that is regulated by SOX2 in hUCB-MSCs.

\section{SOX2 directly binds to the DKK1 promoter region and} activates DKK1 expression. The SOX family proteins possess a DNA-binding HMG domain and additional activation or repression domains that are implicated in transcriptional regulation. ${ }^{31}$ The evolutionarily conserved region of $D K K 1$ was determined using database searches (UCSC genome browser, human genome March 2006 assembly, http://genome.ucsc.edu/). The human genome sequence surrounding the DKK1 transcriptional start site, from $5 \mathrm{kbps}$ upstream to $5 \mathrm{kbps}$ downstream, was searched. We found that the exons and the promoter region around $1 \mathrm{kbps}$ upstream are highly conserved in mammalian genomic DNA (Figure 4a). SOX2 typically binds $5^{\prime}$-CTTTGTT-3' or 5'-CATTGTT-3' sequences, ${ }^{31}$ and one SOX2-binding motif was identified at -76 bps (Figure $4 a$ ). To determine whether SOX2 binds to the DKK1 promoter region, a ChIP assay was performed. Tera-1 was used as a positive control, because SOX2 is highly expressed in tera-1 cells. SOX2 bound $D K K 1$ in sh-control-treated cells. The signal disappeared in sh-SOX2-treated cells, and the same result was observed using siRNA-mediated inhibition (Figure $4 \mathrm{~b}$ and Supplementary Figure 5a).

To examine SOX2 activity at the DKK1 promoter, the human genome fragment from -974 bps to +119 bps around the DKK1 transcription start site was cloned into the luciferase-enhancer vector (Figure $4 \mathrm{c}$ ). This region contains a predicted SOX2-binding site $(\mathrm{B})$, which was confirmed by ChIP. The sh-SOX2 treatment resulted in significantly lower luciferase activity than the sh-control when transfection and luciferase activities were normalized to the SV40-promoter control vector (Figure 4d). To verify these results, SOX2 inhibition by siRNA in hUCB-MSCs was also tested. The siSOX2 treatment resulted in significantly lower luminescence than the si-control (Supplementary Figure $5 b$ ). These results provided evidence that $D K K 1$ expression is positively regulated by SOX2, and that $D K K 1$ is a direct SOX2-target gene.

To confirm the SOX2 binding at the DKK1 promoter, the luciferase assay was performed using mutated constructs (Figure 4e). Plasmid M1 has a single point mutation; the 

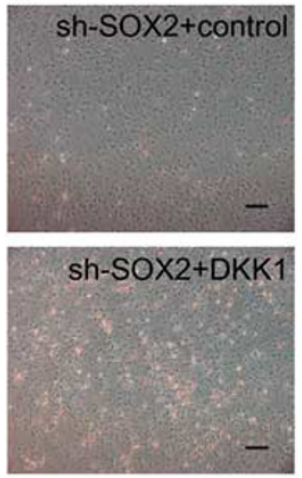

d
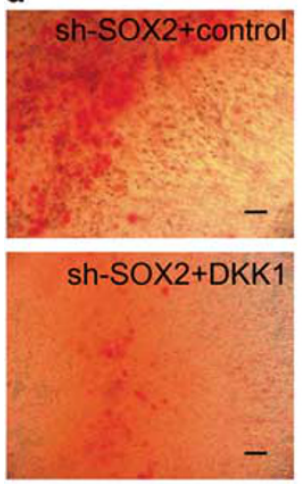

g

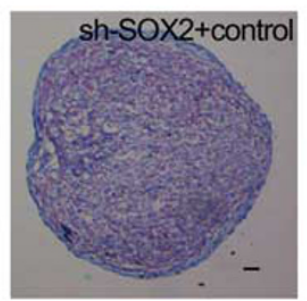

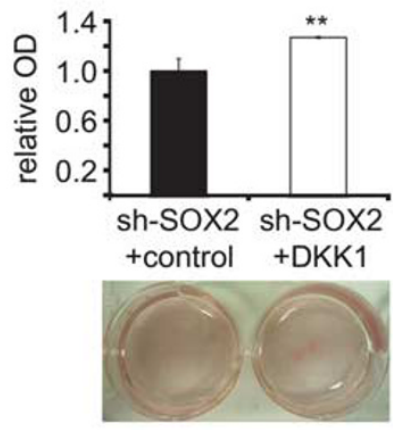

e

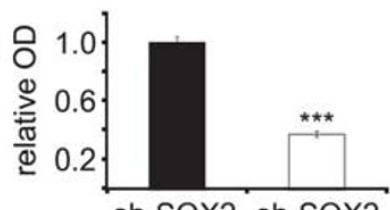

sh-SOX2 sh-SOX2

+control +DKK1

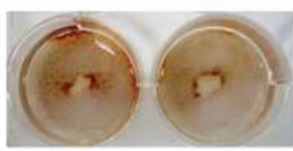

h

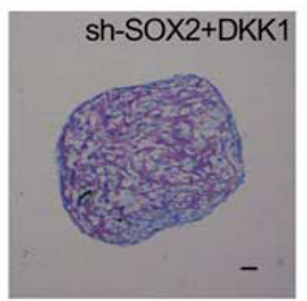

C

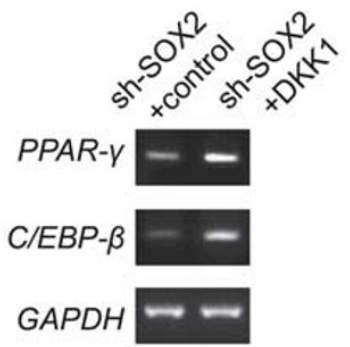

$\mathbf{f}$
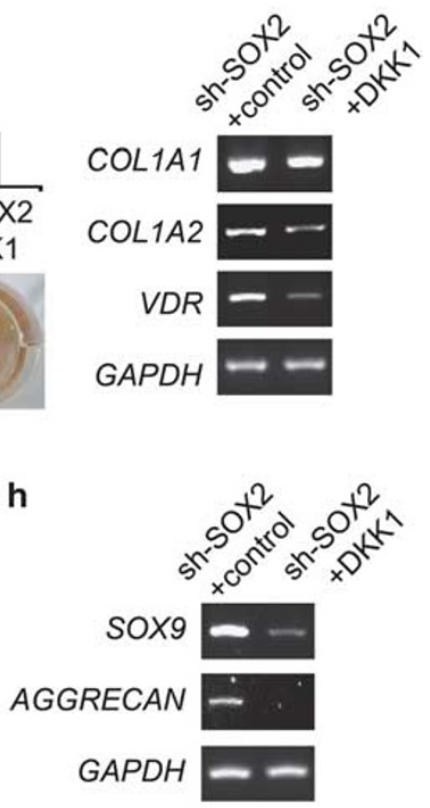

Figure 5 Ectopic expression of DKK1 in SOX2-knockdown hUCB-MSCs reverted the differentiation malformation. (a-c) The adipogenic deformity of lentiviral-mediated SOX2-knockdown hUCB-MSCs was rescued after ectopic expression of DKK1, and this result was confirmed by morphology (a), oil red $\mathrm{O}$ elution (b), and marker gene analysis (c). The scale bar represents $100 \mu \mathrm{m} .{ }^{* \star} P<0.01$. (d-f) The ability of hUCB-MSCs to undergo osteogenic differentiation upon SOX2 knockdown by lentivirus was decreased after the ectopic expression of DKK1, and this result was confirmed by morphology (d), alizarin red S elution (e), and marker gene analysis (f). The scale bar represents $100 \mu \mathrm{m}$. ${ }^{* * *} P<0.001$. ( $\mathbf{g}$ and $\mathbf{h}$ ) The ability of hUCB-MSCs to undergo chondrogenic differentiation upon SOX2 knockdown by lentivirus was decreased by the ectopic expression of DKK1, and this result was confirmed by pellet size (g) and marker gene analysis (h). The scale bar represents $100 \mu \mathrm{m}$

second ' $T$ ' in the HMG domain-binding region was substituted with an ' $A$ '. M1 is also the SOX2-binding sequences. Plasmid $\mathrm{M} 3$ has three point mutations in which the middle 'TGT' was substituted with 'CAG'. Normal hUCB-MSCs and tera-1 cells, which were not treated with lentivirus or siRNA, were used for detecting luciferase activity (Figures $4 \mathrm{f}$ and $\mathrm{g}$ ). M1-transfected cells had similar luminescence activity compared with the nonmutated DKK1 promoter vector-transfected cells. However, M3-transfected hUCB-MSCs and tera-1 cells showed significantly decreased luminescence, compared with the control. These results demonstrated that the binding of SOX2 to the HMG domain of $D K K 1$ directly regulates the transcription of $D K K 1$.
Ectopic expression of DKK1 in SOX2-knockdown hUCBMSCs recovers the differentiation malformations. A DKK1 rescue experiment in SOX2-inhibited hUCB-MSCs was performed to validate the role of DKK1 in SOX2expressing hMSCs. The adipogenic differentiation ability increased after the ectopic expression of DKK1 in sh-SOX2treated cells (Figure 5a). Elevated optical density after elution of oil red $\mathrm{O}$ was also increased by the ectopic expression of DKK1 in sh-SOX2-treated cells (Figure 5b), and the expression of adipogenic marker genes was altered. The expression of $P P A R-\gamma$ and $C / E B P-\beta$ increased after ectopic DKK1 expression in sh-SOX2-treated cells (Figure $5 \mathrm{c}$ ). The osteogenic and chondrogenic ability of SOX2 knockdown 
hUCB-MSCs decreased after the ectopic expression of DKK1 (Figures $5 d-h$ ). The number of alizarin red S-stained cells decreased after ectopic expression of DKK1 in sh-SOX2-treated cells, and these results were confirmed by the elution of alizarin red $S$ (Figures $5 d$ and $e$ ). The expression of osteogenic markers (COL1A2 and VDR) decreased after the ectopic expression of DKK1 in sh-SOX2-treated cells (Figure 5f). Chondrogenesis also decreased after ectopic DKK1 expression in SOX2-knockdown hUCB-MSCs (Figures $5 \mathrm{~g}$ and $\mathrm{h})$. Therefore, DKK1 expression, which is regulated by SOX2, affects the differentiation ability.

C-MYC, not DKK1, is responsible for the proliferation defect of SOX2-inhibited hUCB-MSCs. Cellular proliferation was examined to determine whether DKK1 could rescue not only the differentiation changes, but also the proliferation defect in SOX2-knockdown hUCB-MSCs. However, unlike the differentiation results, the decreased proliferation in shSOX2-treated cells was not recovered by the ectopic expression of DKK1 (Figures 6a and b). Moreover, identical results were obtained using both lentiviral and siRNA inhibition studies (Supplementary Figures $6 a$ and b). To determine whether the addition of WNT ligand could rescue the growth inhibition observed in hUCB-MSCs when SOX2 is downregulated, WNT3A and WNT5A, regarded as canonical and non-canonical WNT ligands, respectively, were added to the growth media. The portion of the SOX2inhibited cells treated with WNT ligand in S phase of the cell cycle increased after $24 \mathrm{~h}$, but there was no change in cell proliferation in response to the WNT3A or WNT5a (Figures $6 \mathrm{c}$, d and Supplementary Figures $6 \mathrm{c}, \mathrm{d}$ ). To confirm the reason of discordance between cell cycle and proliferation test, cell cycle was checked in a time-dependent manner with WNT3A. The results showed that WNT3A influenced cell cycle in a moment, but could not maintain the increased level of $\mathrm{S}$ phase consistently until 3 days (Supplementary Figure 6e).

One of the candidate genes was the transcription factor $c-M Y C$. Although $c-M Y C$ is regulated by $\beta$-CATENIN/TCF signaling, ${ }^{32}$ the expression of $c-M Y C$ was dramatically reduced in SOX2-knockdown hUCB-MSCs (Figure3b and Supplementary Table 2). SOX2-knockdown hUCB-MSCs exhibited decreased c-MYC expression in western blot (Figure 7a and Supplementary Figure 7). To evaluate the role of c-MYC in SOX2-knockdown hUCB-MSCs, enforced expression of $\mathrm{c}-\mathrm{MYC}$ using a retroviral vector was performed in sh-SOX2-treated cells (sh-SOX2 + c-MYC). A GFP expression retrovirus was used as a vector control (VC). To quantify $c-M Y C$ expression, RT-PCR was performed (Figure 7b). The enforced expression of c-MYC in sh-SOX2 cells resulted in higher expression of $c-M Y C$ than in the VC-infected sh-SOX2 cells, and the proliferation was increased after enforced c-MYC expression (Figure 7c). Cell cycle analysis showed that the enforced expression of c-MYC in sh-SOX2 cells caused an increase in S-phase composition (Figure 7d). Moreover, the MTT assay showed that the enforced expression of C-MYC in sh-SOX2 cells resulted in higher proliferation than the VC-infected sh-SOX2 cells (Figure 7e). Therefore, it was suggested that c-MYC expression, regulated by SOX2, was responsible for the growth of hUCB-MSCs. a
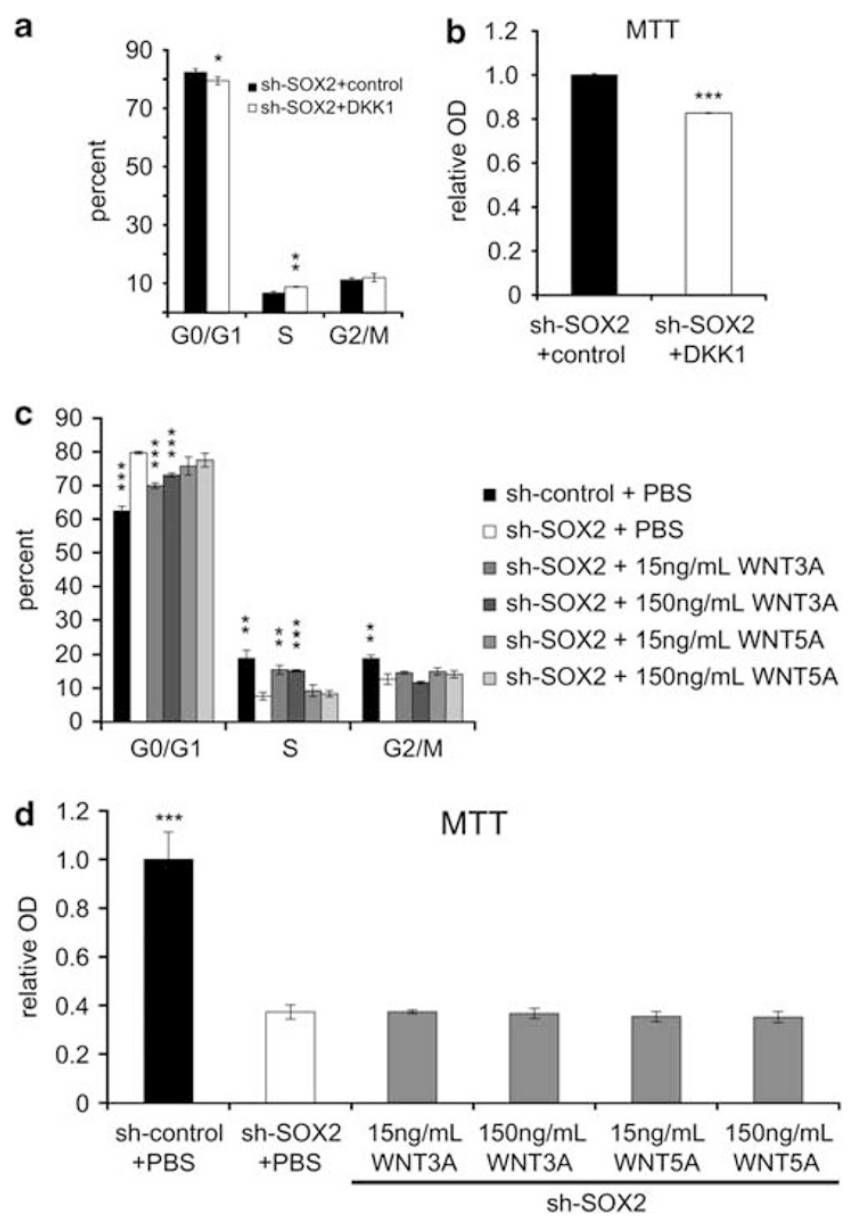

Figure 6 Ectopic expression of DKK1 and WNT ligands cannot recover the proliferation defect caused by SOX2 knockdown in hUCB-MSCs. (a and $\mathbf{b}$ ) Proliferation of hUCB-MSCs with lentiviral SOX2 knockdown did not increase after DKK1 treatment, as confirmed by the MTT assays and FACS analysis. ${ }^{*} P<0.05$; ${ }^{* \star} P<0.01 ;{ }^{* * \star} P<0.001$. (c and d) Treatment with WNT3A and WNT5A could not recover the proliferation defect in SOX2-knockdown hUCB-MSCs. ${ }^{* \star} P<0.01$; ${ }^{\star \star \star} P<0.001$

\section{Discussion}

SOX2 is expressed in hUCB-MSCs although the levels were very low compared with the levels in tera-1 cells. Nevertheless, hUCB-MSCs exhibited stronger expression of SOX2 compared with hBM-MSCs and hAD-MSCs, which have been previously reported to express SOX2., ${ }^{2,33}$ SOX2 inhibition in hMSCs resulted in retarded cell growth, depressed adipogenic differentiation ability, and enhanced osteo-chondrogenic differentiation ability. In hMSCs, SOX2 bound to the promoter region of $D K K 1$ and positively regulated $D K K 1$ transcription. Ectopic expression of DKK1 in SOX2-inhibited hMSCs rescued the differentiation defects, but could not revert the proliferation defects caused by SOX2 inhibition. This proliferation defect was reduced by the additional expression of c-MYC in SOX2-inhibited hMSCs (Figure 8).

The levels of SOX2 expression were variable among the three types of hMSCs. Our previous studies have proven that all three types of hMSCs exhibited self-renewability and multi-lineage differentiation potential. ${ }^{3,14,34}$ There were no 
a

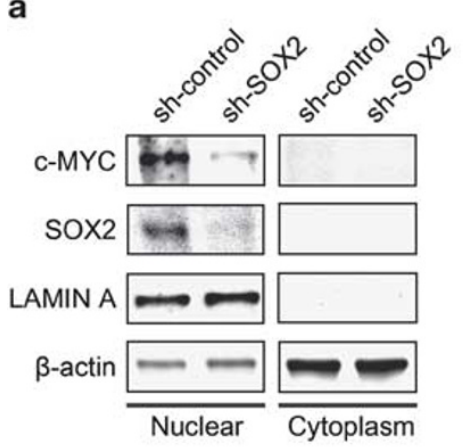

d

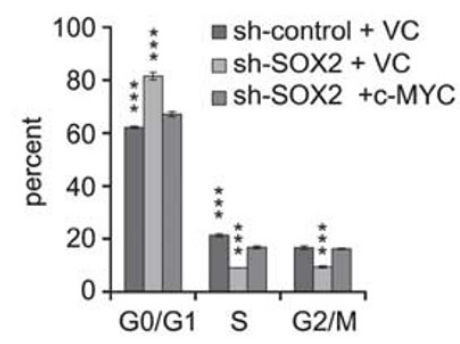

b

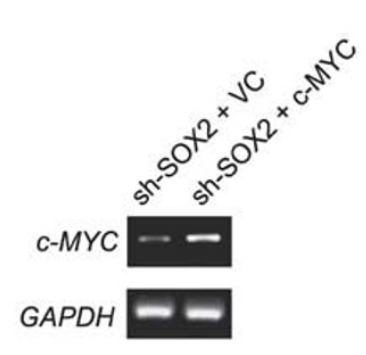

e

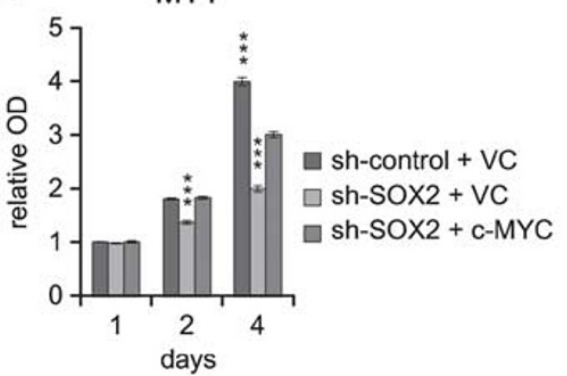

Figure 7 Enforced expression of c-MYC can recover the proliferation defects in SOX2-knockdown hUCB-MSCs. (a) The levels of c-MYC and SOX2 were reduced after SOX2 knockdown. LAMIN A and $\beta$-ACTIN were used as loading controls. (b) The enforced expression of $c-M Y C$ was confirmed by RT-PCR. (c-e) The proliferation of SOX2-knockdown hUCB-MSCs was restored by the addition of C-MYC. The scale bar represents $100 \mu \mathrm{m} .{ }^{* \star *} P<0.001$

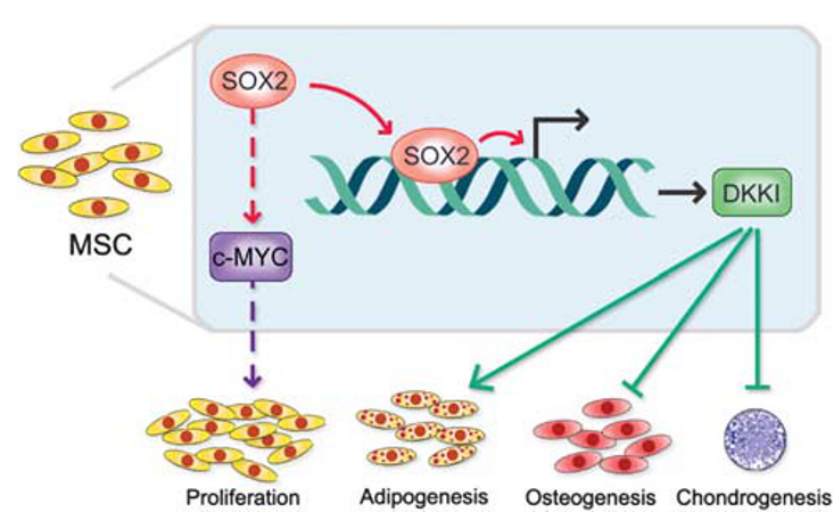

Figure 8 Schematic diagram of proposed mechanism for regulatory roles of SOX2 in hMSCs. SOX2 bound DKK1 promoter region and induced DKK1 transcription. DKK1 regulates differentiation of hMSCs, but could not control proliferation. Proliferation was influenced by c-MYC, which was controlled by SOX2

characteristic differences among these hMSCs. The reason for the differential SOX2 expression might arise from the hMSCs source. The hUCB-MSCs were obtained from the blood of newborns, but the hAD-MSCs and hBM-MSCs were collected from adult donors that were greater than 20 years old. Therefore, the hUCB-MSCs were considered more primitive than the other hMSCs.

The differentiation of hMSCs can be divided into two steps: the differentiation fate-decision step (early) and the fully differentiated step (late). Transient inhibition of SOX2 with siRNA permitted us to determine the effect of SOX2 on the early fate-decision step, and the lentivirus-mediated SOX2 knockdown allowed us to determine the effect of SOX2 on both steps of the hMSC differentiation process. In this study, the differentiation defects observed in SOX2 siRNA-inhibited hMSCs were less severe than the defects observed in hMSCs treated with sh-SOX2 lentivirus. Therefore, the effect of SOX2 on the differentiation of hMSCs occurred during the full differentiation process. Canonical WNT signaling is a mesenchymal regulatory factor that provides instructive cues for the recruitment, maintenance, and differentiation of MSCs. ${ }^{35}$ Once MSCs are committed to the osteogenic lineage, canonical WNT signaling stimulates osteogenic differentiation. Canonical WNT signaling is also likely to promote the initiation of chondrogenesis via crosstalk with TGF- $\beta$ signaling. $^{36}$ WNT/ $\beta$-CATENIN signaling inhibits adipogenic differentiation and enhances chondrogenic differentiation. ${ }^{37}$ Therefore, the decreased adipogenic ability of SOX2-inhibited hMSCs after adipogenic induction was due to the activation of canonical WNT signaling, which occurred through DKK1 suppression in response to SOX2 knockdown.

SOX2 has an essential role in maintaining ESC pluripotency. ${ }^{38}$ A previous study has shown that SOX2-null ESCs differentiated into trophoectoderm-like cells within $72 \mathrm{~h} .^{9}$ These previous results are similar to our current data using hUCB-MSCs in that, SOX2 regulates the differentiation of the stem cells. However, there are several differences between ESCs and hUBC-MSCs. SOX2 is not highly expressed in hUCB-MSCs compared with ESCs. Therefore, SOX2 inhibition alone could not induce the differentiation of hUCB-MSCs. When hUCB-MSCs are induced toward a specific differentiation pathway, the hUCB-MSCs differentiate into the induced lineages specifically. 
SOX2 is also important for ESC proliferation. ${ }^{39}$ SOX2 promotes proliferation by facilitating the $\mathrm{G} 1 / \mathrm{S}$ transition and by transcriptional regulation of the CCND1 gene. ${ }^{23}$ DKK 1 is a negative regulator of the WNT signaling pathway that enhances the proliferation of many types of stem cells. ${ }^{40} \mathrm{In}$ this study, the knockdown of SOX2 in hMSCs caused a reduction in the proliferation rate, although DKK1 levels were also decreased. Ectopic treatment of SOX2-knockdown hMSCs with DKK1 caused more severe proliferation defects. These results indicated that the growth of hUCB-MSCs was controlled by factors beyond WNT signaling. C-MYC is a wellknown transcription factor and serves as a growth regulator in cell lines, including the hMSCs. ${ }^{14}$ The current study provides substantial evidence that c-MYC regulates proliferation and is regulated by SOX2 expression, although it is not clear whether SOX2 directly regulates C-MYC expression.

This study highlights two facets of SOX2 function, which are the differentiation and proliferation of hMSCs. The molecular mechanism by which the loss of SOX2 induces differentiation has been explored in these studies. DKK1 was shown to be a direct transcriptional target of SOX2 in hMSCs, which had never before been determined. The expression of SOX2 in hMSCs is also important for the proliferation and multipotency of hMSCs, largely through the regulation of DKK1 and c-MYC expression, which is quite different from its role in ESCs. This mechanism may be sustained in other ASCs, which exhibited similar levels of SOX2 expression. These results provide a novel function and mechanism for SOX2 in ASC differentiation, and will serve as an important guide for further clinical research on hMSCs and for the developmental study of ASCs.

\section{Materials and Methods}

Cell culture. hUCB-MSCs, hAD-MSCs, and hBM-MSCs were isolated and cultured as previously described. ${ }^{34}$ Briefly, hUCB-MSCs were obtained from umbilical cord blood immediately after full-term delivery with written consent from 20- to 30-year-old mothers. The hUCB-MSCs were maintained in endothelial cell growth medium-2 (Lonza, Basel, Switzerland) containing 10\% fetal bovine serum (FBS). hAD-MSCs were isolated from freshly excised mammary fat tissue acquired from the BaRam Plastic Surgery Hospital. The tissues were obtained from 20- to 30 -year-old women during reduction mammoplasty. The hAD-MSCs were maintained in K-SFM medium supplemented with $2 \mathrm{mM} \mathrm{N}$-acetyl-L-cysteine (Sigma-Aldrich, St. Louis, MO, USA) and $0.2 \mathrm{mM} \mathrm{L-ascorbic} \mathrm{acid} \mathrm{(Sigma-Aldrich).}$ hBM-MSCs were isolated from healthy donors and were cultured in low glucose Dulbecco's modified Eagle's medium (DMEM) supplemented with 10\% FBS, without any additional growth factors. The isolation and research use of hUCBMSCs, hAD-MSCs, and hBM-MSCs were approved by the Boramae Hospital Institutional Review Board with written consent. All procedures were approved by the Institutional Review Board of Seoul National University (hUCB-MSC number 0603/001-002; hAD-MSC number 0600/001-001; hBM-MSC number 0910/001003). The tera- 1 cell line was purchased from ATCC (HTB-105, Manassas, VA, USA) and maintained in DMEM low-glucose medium (Gibco BRL, Grand Island, NY, USA) containing 10\% FBS (Gibco BRL).

Transfection. Lentiviruses were generated using the ViraPower Lentiviral packaging mix (Invitrogen, Carlsbad, CA, USA). Lipofectamine 2000 (Invitrogen) was used to transfect 293FT cells (Invitrogen) with SHDNAC-TRCN0000003253 (sh-SOX2) and SHCOO2 (sh-control, random sequence inserted; Sigma). The cells were transfected with SOX2 short hairpin RNA-producing lentiviruses at an multiplicity of infection of 5-10. Polybrene (Sigma) was added to the cell culture medium at a final concentration of $6 \mu \mathrm{g} / \mathrm{ml}$, and the cell culture medium was replaced with fresh culture medium the day after transfection. For selection, puromycin was added to the culture at a final concentration of $5 \mu \mathrm{g} / \mathrm{ml}$ for 5 days.

Retroviruses were used for c-MYC overexpression. The 293FT (Invitrogen) cells were transfected with VSV-G, gag/pol (Sigma), and the c-MYC expression vector
(c-MYC, pMXs-hc-MYC; Addgene, Cambridge, MA, USA) or the GFP VC (Cell biolabs, San Diego, CA, USA) using Lipofectamine 2000 (Invitrogen). Forty-eight hours later, the supernatant was collected and filtered. The sh-control and sh-SOX2 cells were transfected with virus containing the c-MYC expression or GFP control vector with polybrene at a final concentration of $6 \mu \mathrm{g} / \mathrm{ml}$ (Sigma).

Transient transfection assays were performed using commercially available, specific siRNAs against SOX2 (si-SOX2) and a non-targeting control siRNA (si-control) (ON Target plus SMARTpool, Dharmacon, Lafayette, CO, USA). The siRNA transfections were performed according to the manufacturer's instructions. The cells were incubated with $30 \mathrm{nM}$ siRNA for $48 \mathrm{~h}$. After inhibition, sequential experiments were performed for genetical and characterization analyses.

The DKK1 recovery assay was conducted using a DKK1 plasmid vector (number SC303946; OriGene Technologies, Rockville, MD, USA) and a control vector (number PCMV6XL5; OriGene Technologies). The plasmid transfections were performed according to the manufacturer's instructions. The cells were incubated with $2 \mu \mathrm{g}$ of plasmid per well of a six-well plate for $48 \mathrm{~h}$. After infection, sequential experiments were performed for genetic and differentiation analyses.

RT-PCR and real-time PCR. Total RNA was isolated from cells using TRIzol reagent following the protocol provided by the manufacturer (Invitrogen). Total RNA was used for cDNA synthesis using the Superscript III First-Strand Synthesis System (Invitrogen). For the PCR array, the RT2 Profiler Stem Cell PCR Array (catalog number PAHS-405; SuperArray, Frederick, MD, USA) was used according to the manufacturer's protocol. For RT-PCR, CDNA and primers were combined with a PCR premix (Bioneer, Sung Nam, Republic of Korea). Real-time PCR was performed by mixing CDNA with primers, and Power SYBR Green PCR Master Mix (Applied Biosystems, Foster City, CA). Real-time PCR was performed using an ABI 7500 Real-time PCR System with supplied software (Applied Biosystems), according to the manufacturer's instructions. RNA expression levels were compared after normalization to endogenous GAPDH. The primer sequences used in this study are listed in Supplementary Table 1.

Immunocytochemistry. The cells were fixed in $4 \%$ paraformaldehyde for $10 \mathrm{~min}$ at room temperature. The cells were permeabilized by exposure to $0.5 \%$ Triton X-100 for $10 \mathrm{~min}$ at room temperature and were blocked for $2 \mathrm{~h}$ with $10 \%$ normal goat serum (Zymed, San Francisco, CA, USA) at room temperature. Primary antibodies were used at the manufacturer's recommended dilution in $5 \%$ normal goat serum. Rabbit anti-SOX2 (number ab5603, Millipore, Billerica, MA, USA) and mouse anti-fibronectin (number ab6328; Abcam, Cambridge, MA, USA) were used for immunostaining. Fibronectin staining was utilized for cytological observation. The secondary fluorescence conjugated antibodies, goat anti-mouse Alexa Fluor 488, and goat anti-rabbit Alexa Fluor 594 (Invitrogen), were each used at a 1: 1000 dilution. Hoechst (Sigma-Aldrich) was used for counterstaining.

MTT cell proliferation assay and FACS assay. The proliferation potential was measured using the MTT assay, which is based on the ability of live cells to convert tetrazolium salt into purple formazan. Briefly, the cells $\left(1 \times 10^{4}\right.$ cells per well) were seeded in 24-well microplates in $450 \mu \mathrm{l}$ media. After $48 \mathrm{~h}, 50 \mu \mathrm{l}$ of MTT stock solution ( $5 \mathrm{mg} / \mathrm{ml}$; Sigma) was added to each well, and the plates were further incubated for $4 \mathrm{~h}$ at $37^{\circ} \mathrm{C}$. The supernatant was removed, and $200 \mu \mathrm{l}$ DMSO was added to each well to solubilize the water-insoluble purple formazan crystals. The absorbance at a wavelength of $540 \mathrm{~nm}$ was measured with an EL800 microplate reader (BIO-TEK Instruments, Winooski, VT, USA).

The DNA content was determined by propidium iodide staining intensity. The cells were fixed with ice-cold $70 \%$ ethanol at $4{ }^{\circ} \mathrm{C}$ for $1 \mathrm{~h}$. Following fixation, $50 \mu \mathrm{g} / \mathrm{ml}$ propidium iodide (Sigma-Aldrich) and $100 \mu \mathrm{g} / \mathrm{ml}$ RNase A (Invitrogen) were added at $37^{\circ} \mathrm{C}$ for $1 \mathrm{~h}$. Cell cycle analysis was performed using a FACS Calibur instrument (Becton-Dickinson Pharmingen, San Jose, CA, USA) equipped with ModFit LT (Verity Software House, Topsham, ME, USA).

In-vitro differentiation assays. To induce adipogenic and osteogenic differentiation, hMSCs were treated as previously reported. ${ }^{3}$ Briefly, the cells were treated with an adipogenic induction medium (DMEM supplemented with $10 \%$ FBS, $200 \mu \mathrm{M}$ indomethacin, $1 \mu \mathrm{M}$ dexamethasone, $0.5 \mathrm{mM}$ isobutyl methylxanthine, and $0.5 \mu \mathrm{g} / \mathrm{ml}$ insulin) or an osteogenic induction medium (DMEM containing 10\% FBS, $100 \mathrm{nM}$ dexamethasone, $50 \mu \mathrm{M}$ ascorbic acid 2-phosphate, and $10 \mathrm{mM} \beta$-glycerophosphate; Sigma-Aldrich). After 2 weeks of induction, the cells were stained to confirm that differentiation occurred. For adipogenic differentiation, the cells were stained with oil red O (Sigma-Aldrich). 
Bound stain was solubilized using $100 \%$ isopropanol, and the released solution was measured at $500 \mathrm{~nm}$, using a spectrophotometer. For osteogenic differentiation, alizarin red S staining was performed. The release of solubilized alizarin red $\mathrm{S}$ was measured at $570 \mathrm{~nm}$ using a spectrophotometer. To induce chondrogenic differentiation, $1 \times 10^{5}$ cells were added to a $15 \mathrm{ml}$ polypropylene tube and pelleted by centrifugation. The pellet was cultured at $37^{\circ} \mathrm{C}$ in a $5 \% \mathrm{CO}_{2}$ incubator in $1 \mathrm{ml}$ of chondrocyte differentiation medium (Lonza) for 2 weeks. After 2 weeks, the round pellets were embedded in paraffin and cut into $3 \mu \mathrm{m}$ sections. For histological evaluation, the sections were stained with toluidine blue.

Western blotting. Whole-cell protein lysates were extracted with a solution containing $1 \%$ Triton X-100, $20 \mathrm{mM}$ Tris $\mathrm{HCl}(\mathrm{pH} 8), 137 \mathrm{mM} \mathrm{NaCl}, 10 \%$ glycerol, and $2 \mathrm{mM}$ EDTA (Sigma-Aldrich). Nuclear and cytoplasmic lysates were extracted using NE-PER reagents (Thermo, Rockford, IL, USA). The protein concentrations were determined using a DC assay kit (Bio-Rad, Hercules, CA, USA), and proteins were separated by $10 \%$ SDS-polyacrylamide gel electrophoresis. The separated proteins were then transferred to nitrocellulose membranes at $100 \mathrm{~V}$ and $350 \mathrm{~mA}$ for $2 \mathrm{~h}$, and probed using the following primary antibodies: $\beta$-CATENIN (number 9562; Cell Signaling Technology Inc, Danvers, MA, USA), p- $\beta$-CATENIN (number 9561; Cell Signaling Technology Inc.), and $\beta$-ACTIN (number 3700; Cell Signaling Technology Inc.). Secondary antibodies were used according to the manufacturer's instructions. Secondary-antibody binding was detected using an enhanced chemiluminescence detection kit (Amersham, Piscataway, NJ, USA) according to the manufacturer's instructions. Protein levels were normalized to $\beta$-ACTIN using Image $\mathrm{J}$ analysis software.

ChIP and luciferase reporter assays. ChIP assays were performed according to the manufacturer's protocol (Upstate Biotechnology, Waltham, MA, USA). Chromatin was immunoprecipitated using rabbit anti-human SOX2 antibodies (number ab15830; Abcam). PCR was performed at a final template dilution of $1: 50$. The primer sequences used in this study are supplied in Supplementary Table 1. For the luciferase assays, the DKK1 promoter was cloned into the pGL3-enhancer vector (Promega, Madison, WI, USA), and the pGL3-SV40control vector was used as a transfection control. The luciferase reporter assays were performed according to the manufacturer's instructions. Briefly, $0.75 \mu \mathrm{g}$ of pGL3-enhancer vector containing the DKK1 promoter, or the pGL3-control vector was transfected using Tfx-50 transfection reagent (Promega) at a $2: 1$ ratio of Tfx50:plasmid per well in a 24-well plate. After $48 \mathrm{~h}$ incubation, the cells were treated with Bright-Glo reagent (Promega) for $2 \mathrm{~min}$, and the luminescence of the cell lysate was measured with an infinite M200 pro luminometer (Tecan, Mannedorf, Switzerland). To confirm the luciferase results, the assay was performed using mutated constructs. The SOX2 HMG-binding domain recognition site was mutated with a site-directed mutagenic primer. Briefly, the pGL3-enhancer vector containing the DKK1 promoter was amplified with a mutagenic primer and Pfu DNA polymerase (Qiagen, Hilden, Germany) for 20-25 cycles. The methylated, nonmutated, parental DNA template in the amplified product was digested with Dpn-I. Nicked dsDNA plasmid was transformed into competent cells, and the mutated plasmid was confirmed by sequencing. The sequences of the mutagenic primers are supplied in Supplementary Table 1. The TOP/FOP Flash assays were performed according to the manufacturer's instructions. The cells were transfected with $1 \mu \mathrm{g}$ TOP Flash or $0.24 \mu \mathrm{g}$ FOP Flash plasmid (Millipore), with $0.1 \mu \mathrm{g}$ of renilla luciferase expression plasmid (Millipore) per well, using the Tfx-50 transfection reagent in a 24-well plate. The cells were treated as indicated, and luciferase activity was measured with the Dual-Luciferase reporter assay system (Promega).

Statistical analyses. The change in the $\mathrm{CT}$ of the target genes was calculated as $\triangle C T=$ (CT of target genes) $-(C T$ of GAPDH). The ratio of the target gene to the housekeeping gene was calculated and expressed as $2^{-\Delta C T}$. This ratio was then used to evaluate the expression of each target gene in SOX2-inhibited hUCBMSCs and control hUCB-MSCs. To determine the changes in gene expression, the normalized gene expression of the target genes in SOX2-inhibited hUCB-MSCs was divided by the normalized expression of the same target gene in the control samples. All experiments were conducted in triplicate, and the results are expressed as the mean \pm S.D. Statistical analyses were conducted via analysis of variance, followed by Duncan's multiple range tests or Student's $t$-test. $P<0.05$ was considered to be significant.

\section{Conflict of Interest}

The authors declare no conflict of interest.
Acknowledgements. This work was supported by a National Research Foundation of Korea (NRF) grant, funded by the Korean government (MEST, 20100020265).

1. Chen $X, X u H$, Yuan $P$, Fang F, Huss M, Vega VB et al. Integration of external signaling pathways with the core transcriptional network in embryonic stem cells. Cell 2008; 133: 1106-1117.

2. Riekstina U, Cakstina I, Parfejevs V, Hoogduijn M, Jankovskis G, Muiznieks I et al. Embryonic stem cell marker expression pattern in human mesenchymal stem cells derived from bone marrow, adipose tissue, heart and dermis. Stem Cell Rev 2009; 5: 378-386.

3. Park SB, Yu KR, Jung JW, Lee SR, Roh KH, Seo MS et al. bFGF enhances the IGFsmediated pluripotent and differentiation potentials in multipotent stem cells. Growth Factors 2009; 27: 425-437.

4. Boiani M, Scholer HR. Regulatory networks in embryo-derived pluripotent stem cells. Nat Rev Mol Cell Biol 2005; 6: 872-884.

5. Seo KW, Lee SR, Bhandari DR, Roh KH, Park SB, So AY et al. OCT4A contributes to the stemness and multi-potency of human umbilical cord blood-derived multipotent stem cells (hUCB-MSCs). Biochem Biophys Res Commun 2009; 384: 120-125.

6. Boyer LA, Lee TI, Cole MF, Johnstone SE, Levine SS, Zucker JP et al. Core transcriptional regulatory circuitry in human embryonic stem cells. Cell 2005; 122: 947-956.

7. Loh $\mathrm{YH}, \mathrm{Wu} \mathrm{Q}$, Chew JL, Vega VB, Zhang W, Chen X et al. The Oct4 and Nanog transcription network regulates pluripotency in mouse embryonic stem cells. Nat Genet 2006; 38: 431-440.

8. Ivanova N, Dobrin R, Lu R, Kotenko I, Levorse J, DeCoste $\mathrm{C}$ et al. Dissecting self-renewal in stem cells with RNA interference. Nature 2006; 442: 533-538.

9. Masui S, Nakatake Y, Toyooka Y, Shimosato D, Yagi R, Takahashi K et al. Pluripotency governed by Sox2 via regulation of Oct3/4 expression in mouse embryonic stem cells. Nat Cell Biol 2007; 9: 625-635.

10. Sharov AA, Masui S, Sharova LV, Piao Y, Aiba K, Matoba R et al. Identification of Pou5f1, Sox2, and Nanog downstream target genes with statistical confidence by applying a novel algorithm to time course microarray and genome-wide chromatin immunoprecipitation data. BMC Genomics 2008; 9: 269.

11. Schwartz PH, Bryant PJ, Fuja TJ, Su H, O'Dowd DK, Klassen H. Isolation and characterization of neural progenitor cells from post-mortem human cortex. J Neurosci Res 2003; 74: 838-851.

12. Fang X, Yu W, Li L, Shao J, Zhao N, Chen $Q$ et al. ChIP-seq and functional analysis of the SOX2 gene in colorectal cancers. OMICS 2010; 14: 369-384.

13. Adhikary S, Eilers M. Transcriptional regulation and transformation by Myc proteins. Nat Rev Mol Cell Biol 2005; 6: 635-645.

14. Bhandari DR, Seo KW, Jung JW, Kim HS, Yang SR, Kang SK et al. The regulatory role of C-MYC on HDAC2 and PcG expression in human multipotent stem cells. J Cell Mol Med 2010; 15: 1603-1614.

15. Roy SS, Hsu CH, Wen ZH, Lin CS, Chakraborty C. A hypothetical relationship between the nuclear reprogramming factors for induced pluripotent stem (iPS) cells generationbioinformatic and algorithmic approach. Med Hypotheses 2011; 76: 507-511.

16. Katoh M. WNT signaling in stem cell biology and regenerative medicine. Curr Drug Targets 2008; 9: 565-570.

17. Augello A, De Bari C. The regulation of differentiation in mesenchymal stem cells. Hum Gene Ther 2010; 21: 1226-1238.

18. Gregory CA, Singh H, Perry AS, Prockop DJ. The Wnt signaling inhibitor dickkopf-1 is required for reentry into the cell cycle of human adult stem cells from bone marrow. J Biol Chem 2003; 278: 28067-28078.

19. Qiao L, Xu ZL, Zhao TJ, Ye LH, Zhang XD. Dkk-1 secreted by mesenchymal stem cells inhibits growth of breast cancer cells via depression of Wnt signalling. Cancer Lett 2008; 269: 67-77.

20. Gregory CA, Gunn WG, Reyes E, Smolarz AJ, Munoz J, Spees JL et al. How Wnt signaling affects bone repair by mesenchymal stem cells from the bone marrow. Ann NY Acad Sci 2005; 1049: 97-106.

21. Park JR, Jung JW, Lee YS, Kang KS. The roles of Wnt antagonists Dkk1 and sFRP4 during adipogenesis of human adipose tissue-derived mesenchymal stem cells. Cell Prolif 2008; 41: $859-874$.

22. Kormish JD, Sinner D, Zorn AM. Interactions between SOX factors and Wnt/beta-catenin signaling in development and disease. Dev Dyn 2010; 239: 56-68.

23. Chen Y, Shi L, Zhang L, Li R, Liang J, Yu W et al. The molecular mechanism governing the oncogenic potential of SOX2 in breast cancer. J Biol Chem 2008; 283: 17969-17978.

24. Mansukhani A, Ambrosetti D, Holmes G, Cornivelli L, Basilico C. Sox2 induction by FGF and FGFR2 activating mutations inhibits Wnt signaling and osteoblast differentiation. J Cell Biol 2005; 168: 1065-1076.

25. Basu-Roy U, Ambrosetti D, Favaro R, Nicolis SK, Mansukhani A, Basilico C. The transcription factor Sox2 is required for osteoblast self-renewal. Cell Death Differ 2010; 17: 1345-1353.

26. Episkopou V. SOX2 functions in adult neural stem cells. Trends Neurosci 2005; 28: 219-221.

27. Biernaskie J, Paris M, Morozova O, Fagan BM, Marra M, Pevny L et al. SKPs derive from hair follicle precursors and exhibit properties of adult dermal stem cells. Cell Stem Cell 2009; 5: 610-623. 
28. Conrad S, Renninger M, Hennenlotter J, Wiesner T, Just L, Bonin M et al. Generation of pluripotent stem cells from adult human testis. Nature 2008; 456: 344-349.

29. Shim JH, Cho SA, Seo MJ, Kim JH, Ryu NK, Yoo KH et al. Proteomic analysis of timedependent difference of protein expression profile changes during neuronal differentiation of mouse embryonic stem cells. Mol Cells 2010; 29: 239-244.

30. Menicanin D, Bartold PM, Zannettino AC, Gronthos S. Genomic profiling of mesenchymal stem cells. Stem Cell Rev 2009; 5: 36-50.

31. Kamachi $Y$, Uchikawa M, Kondoh $H$. Pairing SOX off: with partners in the regulation of embryonic development. Trends Genet 2000; 16: 182-187.

32. Chan $E F$, Gat U, McNiff JM, Fuchs E. A common human skin tumour is caused by activating mutations in beta-catenin. Nat Genet 1999; 21: 410-413.

33. Dal Pozzo S, Urbani S, Mazzanti B, Luciani P, Deledda C, Lombardini L et al. High recovery of mesenchymal progenitor cells with non-density gradient separation of human bone marrow. Cytotherapy 2010; 12: 579-586.

34. Bhandari DR, Seo KW, Roh KH, Jung JW, Kang SK, Kang KS. REX-1 expression and p38 MAPK activation status can determine proliferation/differentiation fates in human mesenchymal stem cells. PLoS One 2010; 5: e10493.

35. Baksh D, Tuan RS. Canonical and non-canonical Wnts differentially affect the development potential of primary isolate of human bone marrow mesenchymal stem cells. J Cell Physiol 2007; 212: 817-826.
36. Ling L, Nurcombe V, Cool SM. Wnt signaling controls the fate of mesenchymal stem cells. Gene 2009; 433: 1-7.

37. Kirton JP, Crofts NJ, George SJ, Brennan K, Canfield AE. Wnt/beta-catenin signaling stimulates chondrogenic and inhibits adipogenic differentiation of pericytes: potential relevance to vascular disease? Circ Res 2007; 101: 581-589.

38. Li W, Zhou H, Abujarour R, Zhu S, Young Joo J, Lin T et al. Generation of humaninduced pluripotent stem cells in the absence of exogenous Sox2. Stem Cells 2009; 27: 2992-3000.

39. Wang ZX, Teh CH, Kueh JL, Lufkin T, Robson P, Stanton LW. Oct4 and Sox2 directly regulate expression of another pluripotency transcription factor, Zfp206, in embryonic stem cells. J Biol Chem 2007; 282: 12822-12830.

40. Yi F, Merrill BJ. Stem cells and TCF proteins: a role for beta-catenin-independent functions. Stem Cell Rev 2007; 3: 39-48.

(c) This work is licensed under the Creative Commons Attribution-NonCommercial-No Derivative Works 3.0

Unported License. To view a copy of this license, visit http:// creativecommons.org/licenses/by-nc-nd/3.0

Supplementary Information accompanies the paper on Cell Death and Differentiation website (http://www.nature.com/cdd) 